BULL. AUSTRAL. MATH. SOC.

VOL. $28(1983), 121-129$.

\title{
SUBORDINATION THEOREMS FOR $p$-VALENT FUNCTIONS WITH INITIAL GAPS
}

\section{Prem Singh and A.V.V. Satyanarayana}

In the present note we prove some results for p-valent functions with initial gaps by means of $N$-subordination.

\section{Introduction}

A function $f(z)$ analytic in the unit disc $D:\{z:|z|<1\}$, normalized, so that $f(0)=0, f^{\prime}(0)=1$, is called spiral like if and only if there exists a real number $\alpha$ such that

$$
\operatorname{Re}\left(e^{i \alpha} \frac{z f^{\prime}(z)}{f(z)}\right)>0, z \in D .
$$

The following definition is due to Brickman [1].

An analytic function $f(z), f(0)=0=f^{\prime}(0)-1$ is called $\varphi$-like in $D$ if and only if

$$
\operatorname{Re}\left(\frac{z f^{\prime}(z)}{\varphi(f(z))}\right)>0, \quad z \in D
$$

where $\varphi(w)$ is analytic in $f(D), \varphi(0)=0, \operatorname{Re} \varphi^{\prime}(0)>0$.

The functions $f(z)$ and $F(z)$ are regular in $D$ and $f(0)=F(0)$. Then $f(z)$ is said to be subordinate to $F(z)$, denoted by $f(z)<F(z)$, if there exists a regular function $w(z)$ such that for $z \in D$, $f(z)=F(w(z)),|w(z)|<1$. For $F(z)$ univalent in $D, f(z)<F(z)$ is equivalent to $f(0)=F(0)$ and $f(D) \subset F(D)$.

The following definition of $N$-subordination is due to Singh and Tygel

Received 23 May 1983. 
$[7]$.

DEFINITION A. Let $f(z)$ and $F(z)$ be analytic in $D$. Then $f(z)$ will be called $N$-subordination to $F(z)$ in $D$, denoted by $f(z)<_{N} F(z)$ if and only if there exists a schwartz function $\varphi(z)$ such that

(1) $|\varphi(z)| \leq|z|^{N}, z \in D$, and

(2) $f(z)=E(\varphi(z))$.

For $N=1$, it is an usual subordination, and every normalized univalent starlike function $f(z)$ with initial gap of width $N$, $z f^{\prime}(z) / f(z)<_{N}(1+z) /(1-z)$ holds.

For example, if we take $f(z)=\left(1+z^{N}\right) /\left(1-z^{N}\right)$ and $F(z)=(1+z) /(1-z)$, then $f(z)<_{N} F(z)$. Clearly $f(z)<_{N} F(z)$ implies $f(z)<F(z)$ but not implied by; and $f(z)<{ }_{N} F(z)$ can be written as $f(z)<F\left(z^{N}\right)$.

In this paper we generalize the results of Ruscheweyh [5] for $p$-valent functions with initial gaps by using the new concept of $N$-subordination.

Ruscheweyh [5] proved the following:

THEOREM A. Let $G(z)$ be convex conformal mapping of $D, G(0)=1$ and let

$$
F(z)=z \exp \left(\int_{0}^{z} \frac{G(x)-1}{x} d x\right) .
$$

Let $f(z)$ be analytic in $D, f(0)=0, f^{\prime}(0)=1$. Then

$$
\frac{z f^{\prime}(z)}{f(z)}<G(z), \quad z \in D \text {, }
$$

if and only if, for azz $|s| \leq 1,|t| \leq 1$,

$$
\frac{f(s: i)}{f(t z)}<\frac{F(s z)}{F(t z)}
$$

holds.

The following lemma is due to Lewandowski [2]. 


$$
p \text {-valent functions }
$$

LEMMA A. Let $f(z)$ be analytic in $D, f(0)=0, f^{\prime}(0)=1$. Then we have

$$
\operatorname{Re}\left(e^{i \alpha} \frac{z f^{\prime}(z)}{f(z)}\right)>0, \quad z \in D \text {, }
$$

if and only if there exists numbers $\delta(z)>0, z \in D$, such that, for all $t \in(0, \delta(z))$,

$$
\left|f\left(z\left(1-e^{i \alpha} t\right)\right)\right| \leq|f(z)|, \quad z \in D
$$

We now prove Theorem 1 .

2.

THEOREM 1. Let $G(z)$ be convex conformal map of $D, G(0)=p$,

$$
F(z)=z \exp \left(\int_{0}^{z} \frac{G(x)-p}{x}\right) d x .
$$

Let

$$
f(z)=z^{p}+a_{p+N} z^{p+N}+a_{p+N+1} z^{p+N+1}+\ldots
$$

be analytic in $D$. Then

$$
\frac{z f^{\prime}(z)}{f(z)}<{ }_{N} G(z), \quad z \in D,
$$

if and only if, for alz $|s| \leq 1,|t| \leq 1$,

$$
(t / s)^{p-1} \frac{f(s z)}{f(t z)}<N \frac{F(s z)}{F(t z)}
$$

holds.

Proof. Assume that (2.3) holds. Let $|s| \leq 1,|t| \leq 1, s \neq t$. Then

$$
g(z)=\int_{0}^{z}\left(\frac{s}{1-s x}-\frac{t}{1-t x}\right) d x
$$

is convex and univalent in $D$. By coupling of the generai subordination theorem [6, Theorem 4.1] with the definition of $N$-subordination and (2.3), we can get

$$
\left(\frac{z f^{\prime}(z)}{f(z)}-p\right) * g(z)<_{N}(G(z)-p) * g(z)
$$


where * is the Hadamard product. For every analytic function $h(z)$ with $h(0)=0$, we have

$$
h(z) * g(z)=\int_{t z}^{s z} \frac{h(x)}{x} d x
$$

and thus, from (2.5) and (2.6), we have

$$
\int_{t z}^{s z}\left(\frac{f^{\prime}(x)}{f(x)}-\frac{p}{x}\right) d x<\int_{t z}^{s z} \frac{G(x)-p}{x} d x .
$$

This implies

$$
\exp \left(\int_{t z}^{s z}\left(\frac{f^{\prime}(x)}{f(x)}-\frac{p}{x}\right) d x\right)<_{N} \exp \left(\int_{t z}^{s z} \frac{G(x)-p}{x} d x\right) .
$$

By simplification we can get (2.4).

Conversely, let (2.4) holds. By putting $t=1$ in (2.4) and then for all $|s| \leq 1$,

$$
\frac{f(s z)}{f(z)}=s^{p-1} \frac{F\left(s \varphi_{s}(z)\right)}{F\left(\varphi_{s}(z)\right)}
$$

where $\varphi_{s}(z)$ is analytic in $D,\left|\varphi_{s}(z)\right| \leq|z|^{N}$.

Thus we can find a sequence $s_{k} \rightarrow 1$ such that $\varphi_{s_{k}} \rightarrow \varphi^{*}$ locally uniformally in $D$ where $\left|\varphi^{*}(z)\right| \leq|z|^{N}, z \in D$. Hence, for fixed $z \in D$,

$$
\begin{aligned}
\frac{z f^{\prime}(z)}{f(z)} & =\lim _{k \rightarrow \infty} \frac{f\left(s_{k} z\right)-f(z)}{\left(s_{k}-1\right) f(z)} \\
& =\lim _{k \rightarrow \infty}\left\{s_{k}^{p-1} \frac{\varphi_{s_{k}}(z)}{F\left(\varphi_{s_{k}}(z)\right)} \cdot \frac{F\left(s_{k} \varphi_{s_{k}}(z)\right)-F\left(\varphi_{s_{k}}(z)\right)}{s_{k} \varphi_{s_{k}}(z)-\varphi_{s_{k}}(z)}+\frac{s_{k}^{p-1}-1}{s_{k}-1}\right\} \\
& =\frac{\varphi^{*}(z)}{F\left(\varphi^{*}(z)\right)} \cdot F^{\prime}\left(\varphi^{*}(z)\right)+p-1 .
\end{aligned}
$$

This implies

$$
\text { Range }\left(\frac{z f^{\prime}(z)}{f(z)}\right) \subset \operatorname{Range}\left(\frac{z F^{\prime}(z)}{F(z)}+p-1\right)
$$


Since $z F^{\prime}(z) / F(z)+p-1=G(z)$ is convex conformal by assumption, (2.3) follows, in view of (2.7) and (2.2).

COROLLARY 1. Let $f(z)$ be analytic in $D$ and of the form (2.2). Then

$$
\operatorname{Re}\left(e^{i \alpha} \frac{z f^{\prime}(z)}{f(z)}\right)>\beta \geq 0, z \in D
$$

for certain $\alpha \in(-(\pi / 2), \pi / 2), p \cos \alpha>\beta$, if and only if

$$
(t / s)^{p} \frac{f(s z)}{f(t z)}<{ }_{N}\left(\frac{1-t_{z}^{N}}{1-s_{z}}\right)^{(2 / N)(p \cos \alpha-\beta) \exp (-i \alpha)}
$$

for all $|s| \leq 1,|t| \leq 1$.

Proof. Assume that (2.8) holds. Then we have

$$
\operatorname{Re}\left[\frac{e^{i \alpha}}{p \cos \alpha} \cdot \frac{z f^{\prime}(z)}{f(z)}-\frac{i \sin \alpha}{\cos \alpha}\right]>\frac{\beta}{p \cos \alpha} .
$$

Therefore

$$
\frac{e^{i \alpha}}{p \cos \alpha} \cdot \frac{z f^{\prime}(z)}{f(z)}-\frac{i \sin \alpha}{\cos \alpha}=\frac{1+(1-(2 \beta / p \cos \alpha)) \varphi(z)}{1-\varphi(z)}
$$

for some $\varphi(z)$, which is analytic in $D,|\varphi(z)| \leq|z|^{N}, z \in D$. This implies that the required function $F(z)$ satisfies

$$
\frac{e^{i \alpha}}{p \cos \alpha}\left(\frac{z \dot{F}^{\prime}(z)}{F(z)}+p-1\right)-\frac{i_{\sin \alpha}}{\cos \alpha}=\frac{1+(1-(2 \beta / p \cos \alpha)) z^{N}}{1-z^{N}}
$$

On solving the above equation for $F(z)$ one can easily get

$$
F(z)=z\left(1-z^{N}\right)-(2 / N)(p \cos \alpha-\beta) \exp (-i \alpha)
$$

and the result follows by substituting (2.10) in (2.4).

In Corollary 1 the case $s=1, t=\alpha=\beta=0$, gives

$$
\frac{f(z)}{z^{p}}<N \frac{1}{(1-z)^{(2 p / N)}}
$$

for every p-valent function with initial gaps of the form (2.2). The case $N=p=s=1, t=\alpha=\beta=0$, gives $f(z) / z<1 /(1-z)^{2}$ which is a known result for every normalized starlike function [3]. The case when 
$N=p=s=1, t=\alpha=0, \beta=\frac{1}{2}$, gives $f(z) / z<1 /(1-z)$ for every normalized starlike function of order $\frac{1}{2}[6]$.

The following corollary is an extended version of Lemma A.

COROLLARY 2. Let $f(z)$ be analytic in $D$ and of the form (2.2). Then

$$
\operatorname{Re}\left(e^{i \alpha} \frac{z f^{\prime}(z)}{f(z)}\right)>\beta \geq 0, \quad z \in D,
$$

if and only if $t \in(0,2 \cos \alpha)$,

$$
\left|f\left(z\left(1-e^{i \alpha} t\right)^{1 / N}\right)\right| \leq F(t, \alpha, \beta, p, N)|f(z)|, \quad z \in D,
$$

where

$$
F(t, \alpha, \beta, p, N)=\left|1-e^{i \alpha} t\right|^{p / N}\left(1-\frac{t}{2 \cos \alpha}\right)^{(2 / N) \cos \alpha(\beta-p \cos \alpha)} .
$$

This bound is sharp and one can easily check that $F(t, \alpha, \beta, p, N)<1$ at least for alz $t \in(0, \cos \alpha)$.

Proof. By putting $t=1$ in (2.9), we get

$$
(1 / s)^{p} \frac{f(s z)}{f(z)}<{ }_{N}\left(\frac{1-z}{1-s z}\right)^{(2 / N)(p \cos \alpha-\beta) \exp (-i \alpha)} .
$$

Let $s=\left(1-e^{i \alpha_{t}} t\right)^{1 / N} ;$ we have

$$
\left|f\left(z\left(1-e^{i \alpha_{t}}\right)^{1 / N}\right)\right|=\left|1-e^{i \alpha_{t}}\right|^{p / N}|f(z)|\left|\frac{1-\varphi(z)}{1-\left(1-e^{i \alpha_{t}} t\right) \varphi(z)}\right|^{\lambda}
$$

where $\varphi(z)$ is an analytic function in $D, \lambda=(2 / N) \cos \alpha(p \cos \alpha-\beta)$, $|\varphi(z)| \leq|z|^{N} \leq|z|, z \in D$. By using the properties of bilinear transformation and by majorization we can get the required result. It is also easy to observe that the result is sharp for the function (2.10).

Following is the definition for $p$-valent $N$ - $\varphi$-like functions with respect to $G$.

DEFINITION 1. Let $G(z)$ be a convex conformal map of $D, G(0)=p$ and $f(z)$ be analytic in $D$ and of the form (2.2). Let $\varphi(\omega)$ be analytic in $f(D), \varphi(0)=0, \varphi^{\prime}(0)=1, \varphi(w)=0$ in $f(D) \mid\{0\}$. Then $f(z)$ is called p-valent $N$ - $\varphi$-like function with respect to $G(z)$ 


$$
\text { p-valent functions }
$$

if and only if

$$
\frac{z f^{\prime}(z)}{\varphi(f(z))}<{ }_{N} G(z), \quad z \in D
$$

THEOREM 2. Let $G(z), f(z), \varphi(\omega)$ be as in Definition 1 and let

$$
R(w)=w \exp \left(\int_{0}^{w}\left(\frac{1}{\varphi(x)}-\frac{1}{x}\right) d x\right)
$$

and $F(z)$ as in (2.1). Then $f(z)$ is p-valent $N$-p-like with respect to $G$ if and only if, for all $|s| \leq 1,|t| \leq 1$,

$$
(t / s)^{p-1} \frac{R(f(s z))}{R(f(t z))}<\frac{F(s z)}{F(t z)} .
$$

Proof. Let $f(z)$ be $p$-valent $N$ - $\varphi$-like with respect to $G$. Let

$$
T(z)=z \exp \left[\int_{0}^{z}\left(\frac{f^{\prime}(x)}{\varphi(f(x))}-\frac{p}{x}\right) d x\right] \text {. }
$$

It implies

$$
\frac{z T^{\prime}(z)}{T(z)}=\frac{z f^{\prime}(z)}{\varphi(f(z))}+1-p
$$

From (2.11),

$$
\frac{R^{\prime}(w)}{R(w)}=\frac{1}{\varphi(w)}
$$

Therefore

$$
\begin{aligned}
T(z) & =z \exp \left(\int_{0}^{z}\left(f^{\prime}(x) \cdot \frac{R^{\prime}(f(x))}{R(f(x))}-\frac{p}{x}\right) d x\right) \\
& =z^{1-p} R(f(z))
\end{aligned}
$$

and

$$
\frac{T(s z)}{T(t z)}=(t / s)^{p-1} \frac{R(f(s z))}{R(f(t z))}
$$

But

$$
\frac{z f^{\prime}(z)}{\varphi(f(z))}<N G(z)
$$

implies that 


$$
\frac{z T^{\prime}(z)}{T(z)}+p-1<_{N} G(z)
$$

By Theorem 1,

$$
\frac{T(s z)}{T(t z)}<_{N} \frac{F(s z)}{F(t z)}
$$

From (2.14) and (2.16) we can calculate (2.12).

Conversely, let (2.12) hold for all $|s| \leq 1,|t| \leq 1$. Then we have (2.13) and (2.14). The required result immediately follows from $(2.13),(2.14)$ and Theorem 1 .

3.

In this section we present a $N$-subordination theorem which is valid for all p-valent functions.

THEOREM 3. Let $f(z)$ be analytic and p-valent in $D$. Then, for $0 \leq t \leq 1$,

$$
\left[\frac{(1+t)^{2}}{4 t}\right]^{p} f\left(t z^{N}\right)<f(z), \quad z \in D .
$$

Proof. From the hypothesis and due to Lemma 1 [4], $f(z)$ has a representation of the form

$$
f(z)=(\varphi(z))^{p}
$$

where $\varphi(z)$ is univalent in $D$. For $\varphi(z)$,

$$
\frac{(1+t)^{2}}{4 t} \varphi(t z)<\varphi(z), z \in D,
$$

follows from Theorem $3[5]$.

From (3.3) we have

$$
\frac{(1+t)^{2}}{4 t} \varphi\left(t z^{N}\right)<_{N} \varphi(z), \quad z \in D .
$$

Thus

$$
\left[\frac{(1+t)^{2}}{4 t}\right]^{p}\left(\varphi\left(t z^{N}\right)\right)^{p}<_{N}(\varphi(z))^{p}, z \in D
$$


The result follows from (3.4) and (3.2).

\section{References}

[1] Louis Brickman, "\$-like analytic functions. I", Bull. Amer. Math. Soc. 79 (1973), 555-558.

[2] Zdzislaw Lewandowski, "Some remarks on a paper of M.S. Robertson", Ann. Univ. Mariae Curie-SkZodowska Sect. A 17 (1963), 43-46 (1965).

[3] Arnold Marx, "Untersuchungen über schlichte Abbildungen", Math. Ann. 107 (1933), 40-67.

[4] M.S. Robertson, "The partial sums of multivalently star-like functions", Ann. of Math. 42 (1941), 829-838.

[5] Stephan Ruscheweyh, "A subordination theorem for $\Phi$-like functions", J. London Math. Soc. (2) 13 (1976), 275-280.

[6] St. Ruscheweyh and T. Sheil-Small, "Hadamard products of Schlicht functions and the Polya-Schoenberg conjecture", Comment. Math. Helv. 48 (1973), 119-135.

[7] Prem Singh and Martin Tygel, "On the coefficient bounds of certain univalent functions", submitted.

Department of Mathematics,

Indian Institute of Technology Kanpur,

IIT Post Office,

Kanpur - 208016 ,

U.P.,

India. 